\title{
Traumatic axonal injury on clinical MRI: association with the Glasgow Coma Scale score at scene of injury or at admission and prolonged posttraumatic amnesia
}

\author{
Hans Kristian Moe, MD, PhD, ${ }^{1}$ Turid Follestad, MSc, PhD, ${ }^{2}$ Nada Andelic, MD, PhD, 3,4 \\ Asta Kristine Håberg, MD, PhD, ${ }^{1,5}$ Anne-Mari Holte Flusund, MD, ${ }^{1,6}$ Kjell Arne Kvistad, MD, PhD, ${ }^{5}$ \\ Elin Hildrum Saksvoll, MD, ${ }^{7}$ Øystein Olsen, MD, ${ }^{7}$ Sebastian Abel-Grüner, MD, ${ }^{5}$ \\ Oddrun Sandrød, MSc, ${ }^{8}$ Toril Skandsen, MD, PhD, ${ }^{1,9}$ Anne Vik, MD, PhD, ${ }^{1,10}$ and \\ Kent Gøran Moen, MD, PhD ${ }^{1,7}$
}

\begin{abstract}
Departments of ${ }^{1}$ Neuromedicine and Movement Science and ${ }^{2}$ Public Health and Nursing, Faculty of Medicine and Health Sciences, Norwegian University of Science and Technology (NTNU), Trondheim; ${ }^{3}$ nstitute of Health and Society, Research Centre for Habilitation and Rehabilitation Models and Services (CHARM), Faculty of Medicine, University of Oslo; " $D$ Department of Physical Medicine and Rehabilitation, Oslo University Hospital, Ullevål; Departments of ${ }^{5}$ Radiology and Nuclear Medicine, ${ }^{8}$ Anesthesiology and Intensive Care Medicine, ${ }^{9}$ Physical Medicine and Rehabilitation, and ${ }^{10}$ Neurosurgery, St. Olav's Hospital, Trondheim University Hospital, Trondheim; ' ${ }^{2}$ epartment of Radiology, Molde Hospital, Molde; and 'Department of Radiology, Levanger Hospital, Nord-Trøndelag Hospital Trust, Levanger, Norway
\end{abstract}

OBJECTIVE The aim in this study was to investigate if MRI findings of traumatic axonal injury (TAI) after traumatic brain injury (TBI) are related to the admission Glasgow Coma Scale (GCS) score and prolonged duration of posttraumatic amnesia (PTA).

METHODS A total of 490 patients with mild to severe TBI underwent brain MRI within 6 weeks of injury (mild TBI: median 2 days; moderate to severe TBI: median 8 days). The location of TAl lesions and measures of total TAl lesion burden (number and volume of lesions on FLAIR and diffusion-weighted imaging and number of lesions on T2*-weighted gradient echo or susceptibility-weighted imaging) were quantified in a blinded manner for clinical information. The volume of contusions on FLAIR was likewise recorded. Associations between GCS score and the location and burden of TAI lesions were examined with multiple linear regression, adjusted for age, Marshall CT score (which includes compression of basal cisterns, midline shift, and mass lesions), and alcohol intoxication. The predictive value of TAI lesion location and burden for duration of PTA > 28 days was analyzed with multiple logistic regression, adjusted for age and Marshall CT score. Complete-case analyses of patients with TAI were used for the regression analyses of GCS scores $(n=268)$ and $\operatorname{PTA}(n=252)$.

RESULTS TAI lesions were observed in $58 \%$ of patients: in $7 \%$ of mild, $69 \%$ of moderate, and $93 \%$ of severe TBI cases. The TAI lesion location associated with the lowest GCS scores were bilateral lesions in the brainstem (mean difference in GCS score -2.5), followed by lesions bilaterally in the thalamus, unilaterally in the brainstem, and lesions in the splenium. The volume of TAI on FLAIR was the measure of total lesion burden most strongly associated with the GCS score. Bilateral TAl lesions in the thalamus had the largest predictive value for PTA > 28 days (OR 16.2, 95\% $\mathrm{Cl}$ 3.9-87.4). Of the measures of total TAI lesion burden, the FLAIR volume of TAI predicted PTA > 28 days the best.

CONCLUSIONS Bilateral TAI lesions in the brainstem and thalamus, as well as the total volume of TAI lesions on FLAIR, had the strongest association with the GCS score and prolonged PTA. The current study proposes a first step toward a modified classification of TAl, with grades ranked according to their relation to these two measures of clinical TBI severity. https://thejns.org/doi/abs/10.3171/2020.6.JNS20112

KEYWORDS craniocerebral trauma; diffuse axonal injury; consciousness disorders; posttraumatic amnesia; neuroimaging; traumatic brain injury

ABBREVIATIONS AIC = Akaike information criterion; $A U C=$ area under the ROC curve; DWI = diffusion-weighted imaging; GCS = Glasgow Coma Scale; GRE = gradient echo; ICC = intraclass correlation coefficient; PTA = posttraumatic amnesia; $R O C=$ receiver operating characteristic; SWI = susceptibility-weighted imaging; TAI = traumatic axonal injury; $\mathrm{TBI}=$ traumatic brain injury.

SUBMITTED January 16, 2020. ACCEPTED June 8, 2020.

INCLUDE WHEN CITING Published online October 23, 2020; DOI: 10.3171/2020.6.JNS20112. 
$\mathrm{M}$ RI is increasingly used as a diagnostic tool after traumatic brain injury (TBI). While CT remains the primary imaging modality to assess intracranial hematomas and hemorrhagic contusions, MRI has a higher sensitivity for parenchymal lesions such as traumatic axonal injury (TAI) and nonhemorrhagic cortical contusions. ${ }^{1,2}$ TAI, also known as diffuse axonal injury, occurs after all severities of TBI and is associated with functional disability. ${ }^{3,4}$ Findings of TAI in the hemispheres or cerebellum on MRI have been described as TAI grade 1, in the corpus callosum as grade 2, and in the brainstem as grade $3 ., 5$ Although not incorporated in this grading, TAI can also be found in deep gray matter such as the thalamus or basal ganglia. ${ }^{6,7}$ Advances in MRI technology and emerging possibilities of artificial intelligence for quantitative and detailed image reading calls for the development of a more comprehensive approach to evaluating TAI lesions on MRI.

The clinical injury severity in the acute phase is usually classified according to the level of consciousness measured by the Glasgow Coma Scale (GCS). ${ }^{8}$ The GCS score has been shown to be an important predictor of functional outcome $^{9}$ and still decisive for the timing of diagnostic procedures and choice of treatment in the management of TBI. The duration of posttraumatic amnesia (PTA) has also been used as an index of injury severity. ${ }^{10}$ PTA represents a period of disorientation, impaired memory, and neurobehavioral disturbances in the acute and subacute phase after TBI, and is an important predictor of functional outcome and time to return to work..$^{10}$ Patients in PTA need close supervision and specialized care in suitable locations. ${ }^{11}$ Still, little is known about the neuroanatomical basis for the prolonged duration of PTA observed in some patients.

A comprehensive characterization of TBI, its initial severity, and different pathoanatomical entities is a key step in achieving precision-medicine approaches to the management of TBI. ${ }^{12,13}$ Although TAI lesions can be described in increasing detail on clinical MRI, to date little is known of their relation to injury severity and early clinical recovery of TBI. Novel insights into the abovementioned relationships may help physicians in setting appropriate goals for treatment and early rehabilitation and to provide realistic outcome expectations.

Hence, this study aimed to investigate how detailed information about TAI lesions on clinical MRI were related to the admission GCS score and prolonged duration of PTA. By using multivariable analyses in a large cohort of patients, the association to both the location and burden of TAI lesions after controlling for other factors could be examined. This unique explorative study forms part of the larger TAI-MRI project and is the first step toward the development of an improved classification system for TAI.

\section{Methods}

\section{Three Cohorts of TBI Patients}

Patients were selected from the following three prospective cohorts: 1) the Trondheim moderate and severe TBI cohort (Trondheim MS-TBI cohort); ${ }^{4}$ 2) the Trondheim mild TBI follow-up study (Trondheim m-TBI co- hort) ${ }^{14}$ and 3) the Oslo severe TBI cohort (Oslo S-TBI cohort)..$^{15}$

The Trondheim MS-TBI cohort consisted of all patients admitted to St. Olav's Hospital, Trondheim University Hospital, between October 2004 and October 2017 with moderate TBI (GCS scores 9-13 or GCS score 14 or 15 and loss of consciousness $>5$ minutes [6 patients]) or severe TBI $(\mathrm{GCS} \leq 8)$ according to the Head Injury Severity Scale. The Trondheim m-TBI cohort consisted of patients aged 16-60 years who presented with mild TBI in the hospital emergency department or the municipal outpatient emergency department between April 2014 and December 2015 and who underwent 3T MRI within 72 hours of injury. Mild TBI was defined according to the WHO criteria: GCS scores 13-15, loss of consciousness < 30 minutes, and PTA $<24$ hours. ${ }^{14}$ The Oslo S-TBI cohort consisted of all patients older than 16 years with a GCS score $\leq 8$ at some time point within the first 24 hours admitted to Oslo University Hospital between January 2009 and January 2011 as part of a multicenter study on severe TBI. ${ }^{15}$

\section{Exclusion Criteria}

Exclusion criteria for this study were 1) age $\geq 70$ years, to reduce the prevalence of age-related nonspecific white matter hyperintensities, ${ }^{16}$ and age $<5$ years, because of low reliability of the GCS score in preschool children; ${ }^{8} 2$ ) MRI performed $>6$ weeks postinjury, due to the attenuation of TAI lesions on MRI over time; ${ }^{17}$ 3) unreadable MRI studies due to large artifacts, more than one missing MRI sequence (see MRI and Image Reading), or acute ischemia/infarction in large-vessel territories on diffusionweighted MRI; and 4) inclusion in the CENTER-TBI project, ${ }^{18}$ because it will form part of the validation cohort for the future revised TAI classification. The final study sample contained 490 patients (Fig. 1).

\section{GCS Score and PTA}

GCS scores were registered at admission (postresuscitation) or, for intubated patients, before sedation (missing: $n=13$ ). For the Trondheim MS-TBI and Oslo S-TBI cohorts, GCS scores were collected at the scene of injury by anesthesiologists $(n=153)$, at the primary hospital by consultants/residents in general surgery $(n=43)$, or at admission to the university hospital $(n=132)$ by consultants or residents in neurosurgery, neurology, or trauma surgery. Excluding patients aged $>70$ years limited the error introduced by dementia. Since alcohol intoxication can affect GCS scores, it was included as a covariate in the regression analyses. The same researchers defined the GCS variable in all three cohorts, limiting the variability. For the Trondheim m-TBI cohort, GCS scores were observed by the study personnel or recorded from the medical record. If lacking, history and clinical descriptions from medical records were used to estimate scores $(n=8)$. For the final study sample, TBI was classified solely according to the GCS score as mild (score 14 or 15), moderate (scores 9-13), and severe (scores 3-8). The duration of PTA was based on prospective measurements using the Galveston Orientation and Amnesia Test or the Orientation $\log ^{19}$ at transfer to rehabilitation units if the patient remained in 


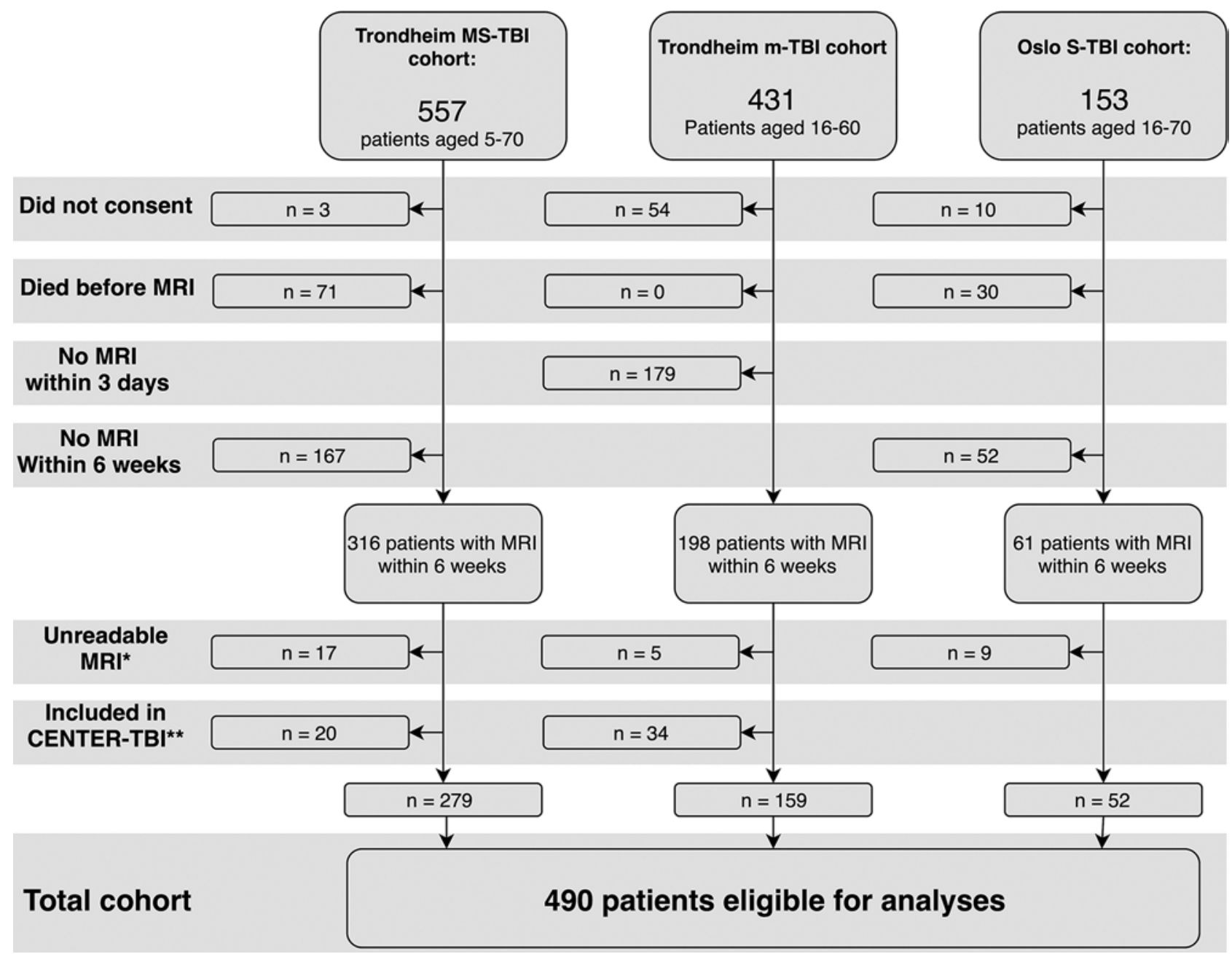

FIG. 1. Flowchart of the 490 included patients. *Unreadable due to poor quality, large artifacts, extensive large-vessel ischemia, or more than one MRI sequence was missing. ${ }^{* *}$ These excluded patients would later serve as part of a validation cohort for the planned modified TAI-MRI classification scheme.

PTA. Otherwise, recorded clinical assessment or interviews with patients and relatives were used. The duration of PTA was recorded as 7-day intervals and $>28$ days (missing: $\mathrm{n}=27$ ).

\section{Other Injury Variables and Classification of CT Findings}

Pupillary dilatation at admission, hypoxia $\left(\mathrm{SaO}_{2}<\right.$ 90\%-92\%), and hypotension (systolic blood pressure < $90 \mathrm{~mm} \mathrm{Hg}$ ) noted before or at admission were recorded. Alcohol intoxication was recorded when biochemically verified, patient disclosed, or clinically suspected. Admission CT scans were assigned a Marshall CT score ${ }^{20}$ by radiologists or residents in radiology in collaboration with neuroradiologists (Trondheim MS-TBI cohort), by reviewing the radiology report (Trondheim m-TBI cohort), or by neuroradiologists (Oslo S-TBI cohort). Scores of 3 or 4 indicate compressed basal cisterns or midline shift, and scores of 5 or 6 indicate intracranial mass lesion (hematoma evacuated at any time point or nonevacuated hematomas $>25 \mathrm{ml}$ ).

\section{MRI and Image Reading}

MRI was performed at 3T $(\mathrm{n}=179), 1.5 \mathrm{~T}(\mathrm{n}=304)$, or 1T $(\mathrm{n}=7)$ at St. Olav's Hospital, Trondheim University Hospital, Oslo University Hospital, or one of their referral hospitals. The MRI protocols are presented in Supplemental Table 1 and previous studies. ${ }^{2,4}$ Patients with more than one missing or unreadable scan of FLAIR, diffusionweighted imaging (DWI), and either T2*-weighted gradient echo (GRE) or susceptibility-weighted imaging (SWI) were excluded (Fig. 1). A total of 488 patients had FLAIR, 485 had DWI, 270 had GRE, and 206 had SWI (98\% of mild TBI had SWI) scans.

All scans were scored blinded for clinical information by consultants in radiology (K.G.M., A.M.H.F., E.S., Ø.O., and S.A.G.), and according to a template developed by the ERA-NET NEURON TAI-MRI partners (project: https:// www.neuron-eranet.eu/_media/TAI-MRI.pdf). The standard TAI grade ${ }^{1}$ and the presence of TAI lesions in each brain region were recorded based on all available sequences. For the basal ganglia, thalamus, and brainstem, TAI 
TABLE 1. Patient characteristics, CT, and MRI findings by TBI injury severity

\begin{tabular}{|c|c|c|c|}
\hline Variable & Severe TBI (GCS 3-8) & Moderate TBI (GCS 9-13) & Mild TBI (GCS 14 or 15) \\
\hline No. of patients & 186 & 142 & 162 \\
\hline Age in yrs, median (IQR) & $27(20-42)$ & $32(21-52)$ & $29(22-44)$ \\
\hline Male/female (\% male) & 153/33 (82) & $102 / 40(72)$ & $107 / 55(66)$ \\
\hline \multicolumn{4}{|l|}{ Injury mechanism } \\
\hline Road traffic accident & $108(58)$ & $61(43)$ & $43(27)$ \\
\hline Fall & $62(33)$ & $57(40)$ & $69(43)$ \\
\hline Struck object & $4(2)$ & $1(1)$ & $17(10)$ \\
\hline Violence & $9(5)$ & $7(5)$ & $23(14)$ \\
\hline Other & $2(1)$ & $12(8)$ & $8(5)$ \\
\hline Unknown & $1(1)$ & $4(3)$ & $2(1)$ \\
\hline GCS score, median (IQR) & $6(4-7)$ & $12(10-13)$ & $15(15-15)$ \\
\hline Hypoxia & $67(36)$ & $11(8)$ & $0(0)$ \\
\hline Hypotension & $48(26)$ & $11(8)$ & $0(0)$ \\
\hline \multicolumn{4}{|l|}{ Pupil dilatation at admission } \\
\hline Unilateral & $41(22)$ & $4(3)$ & $0(0)$ \\
\hline Bilateral & $10(5)$ & $1(1)$ & $0(0)$ \\
\hline Alcohol intoxication at admission* & $64(37)$ & $41(29)$ & $75(47)$ \\
\hline Intubated at scene of injury & $117(63)$ & $26(18)$ & $1(1)$ \\
\hline \multicolumn{4}{|l|}{ PTA in days $†$} \\
\hline $0-7$ & $33(19)$ & $93(72)$ & $160(99)$ \\
\hline $8-14$ & $21(12)$ & $13(10)$ & 0 \\
\hline $15-21$ & $19(11)$ & $9(7)$ & $2(1)$ \\
\hline $22-28$ & $19(11)$ & $5(4)$ & 0 \\
\hline$>28$ & $79(46)$ & $10(8)$ & 0 \\
\hline \multicolumn{4}{|l|}{ Marshall CT score $\ddagger$} \\
\hline 1 & $20(11)$ & $24(17)$ & $117(89)$ \\
\hline 2 & $86(46)$ & $81(57)$ & $10(8)$ \\
\hline 3 or 4 & $36(19)$ & $12(8)$ & $1(1)$ \\
\hline 5 or 6 & $44(24)$ & $25(18)$ & $3(2)$ \\
\hline Evacuated hematoma & $42(23)$ & $22(15)$ & $2(1)$ \\
\hline TAl location on MRI & $173(93)$ & $98(69)$ & $11(7)$ \\
\hline Hemispheres & $160(86)$ & $96(68)$ & $10(6)$ \\
\hline Corpus callosum & $112(60)$ & $39(27)$ & $2(1)$ \\
\hline Basal ganglia & $43(23)$ & $21(15)$ & 0 \\
\hline Thalamus & $55(30)$ & $13(9)$ & 0 \\
\hline Brainstem & $74(40)$ & $17(12)$ & 0 \\
\hline Mesencephalon & $66(35)$ & $16(11)$ & \\
\hline Pons & $34(18)$ & $5(4)$ & \\
\hline Medulla oblongata & $2(1)$ & 0 & \\
\hline Contusions on MRI§ & $145(78)$ & $102(72)$ & $11(7)$ \\
\hline
\end{tabular}

Values are presented as number (\%) unless otherwise indicated.

${ }^{*}$ Positive blood alcohol content and/or clinical suspicion or confirmation; 17 patients had missing information.

† Information on PTA was available for 171 patients in the severe TBI group and 130 patients in the moderate TBI group.

‡ Marshall scores were available for 131 patients in the mild TBI group.

$\S$ Also includes traumatic parenchymal hematomas.

lesions were also classified as unilateral or bilateral. The number of TAI lesions in 58 brain regions were recorded for FLAIR, DWI, and T2*-weighted GRE/SWI sequences. If the count exceeded 10 within one region, a value 15 was assigned for that specific region. ${ }^{17}$ The volumes of TAI lesions on FLAIR and DWI were manually delineated and then calculated using 3D Slicer version 4.8.0. These numbers and volumes of TAI lesions, summarized for each sequence, constituted the different measures of total TAI lesion burden. 
The total volume of cortical contusions and traumatic parenchymal hematomas were combined, hereafter referred to as contusions. Incidental white matter hyperintensities on FLAIR, with typical periventricular distribution ("caps and bands") or in deep white matter, were considered unrelated to trauma and not included as traumatic lesion burden. ${ }^{21}$ Information from all available sequences was used to classify lesions. More details on the MRI scoring is presented in the Supplemental Text.

\section{Interrater Agreement for Traumatic Axonal Injury}

A total of 30 MRI scans scored by K.G.M. were randomly selected from the Trondheim MS-TBI cohort and were independently rescored by Ø.O., A.M.H.F., and E.S. Agreement was assessed for the presence of TAI, TAI grade, total number of TAI on T2*-weighted GRE/SWI, and total volume of TAI on FLAIR and DWI, and of contusions. Interrater reliability for the detection of TAI in the Trondheim m-TBI cohort was presented in a previous paper (Cohen's $\kappa$ 0.58, discordance in $7 / 82$ pairs). ${ }^{2}$

\section{Statistical Analysis}

Patient and injury characteristics are presented as frequencies and percentages or medians with IQR (25th-75th percentiles). Chi-square tests were used for the comparison of proportions and Spearman's rank correlation coefficient $\left(r_{s}\right)$ was used for correlations.

Associations between the MRI variables and the GCS score or PTA (dichotomized at 28 days $^{22}$ ) were analyzed by linear and logistic regressions, respectively. Only patients with at least one TAI lesion on one MRI sequence were included in the regression analyses due to the large proportion of mild TBI patients without MRI findings. The number and volume of lesions were log-transformed using the natural logarithm before being included as covariates in the regression models. Lesion burden values of 0 could not be log-transformed, so a dichotomous variable indicating the presence or absence of TAI was included. Multiple regression analyses were adjusted for age and Marshall CT score (collapsed into four levels [scores 1, 2,3 or 4 , and 5 or 6] and analyzed as a nominal variable) both in models of GCS score and PTA, and also for alcohol intoxication in models of the GCS score.

QQ-plots were used to assess linear regression models for normally distributed residuals. The Akaike information criterion (AIC; lower values indicate a better fit), adjusted $\mathrm{R}^{2}$ (linear regression) and McFadden's pseudo $\mathrm{R}^{2}$ (logistic regression) assessed model fit. Receiver operating characteristic (ROC) curves and DeLong's test for differences in area under the ROC curves (AUCs) were used to compare different models for PTA > 28 days. Completecase analyses for patients with TAI were used for the regression analyses (GCS models: $\mathrm{n}=268$; PTA models: $\mathrm{n}$ $=252$ ). Due to collinearity, no more than one measure of total TAI lesion burden was entered in each regression model.

Interrater reliability for the presence or absence of TAI was evaluated with specific positive and negative agreement. ${ }^{23}$ The intraclass correlation coefficient (ICC; twoway random-effects model for agreement) measured the interrater reliability for ordinal and continuous variables. All counts/volumes were analyzed as $\ln ($ value +1$)$ because a normal assumption is made when calculating ICC. ICC $<0.40$ indicated poor, $0.40-0.59$ fair, $0.60-0.74$ good, and $0.75-1$ excellent agreement; ${ }^{24} \mathrm{p}<0.05$ was considered statistically significant. The statistical analyses were performed using R version 3.5.1 (https://www.r-project.org/).

\section{Ethics}

The study was approved by the Regional Committee for Medical Research Ethics and informed consent was obtained from the patients, or, when incapacitated or younger than 16 years, their next of kin.

\section{Results \\ Demographics, Injury Characteristics, and MRI Findings}

Included patients did not differ from excluded patients with regard to sex or GCS score, but included patients were younger $(p<0.001)$. Patient and injury characteristics for the MRI cohort are presented in Table 1. Patients with mild TBI underwent MRI at a median of 2 days (IQR 2-3 days), moderate TBI at a median of 6 days (IQR 3-14 days, $\mathrm{p}<0.001$ ), and severe TBI at a median of 9 days (IQR 5-17 days, $\mathrm{p}=0.011$ ) postinjury. TAI was present in $58 \%$ of patients (282/490), and contusions were present on MRI in 53\% of patients. The total volume of TAI lesions on FLAIR and DWI were strongly correlated $\left(r_{s}=0.78\right.$; Supplemental Table 2).

\section{Occurrence of TAI, TAI Lesion Location, and GCS Scores}

The proportions of patients with TAI for each GCS score are presented in Fig. 2, and differed between patients with mild (7\%), moderate (69\%), and severe $(93 \%)$ TBI $(\mathrm{p}<0.001)$ (Table 1). The strongest association between TAI and the GCS score (i.e., largest estimated effect in the analyses), irrespective of MRI sequence, was found for bilateral lesions in the brainstem (mean difference in GCS score -2.5$)$, followed by bilateral thalamic lesions $(-1.6)$, unilateral brainstem lesions (-1.6), and lesions in the corpus callosum (-1.5) (Table 2). The association remained high for brainstem lesions after adjusting for the total TAI lesion burden (volume of TAI lesions on FLAIR).

Subsequently, the different main locations of TAI lesions were further divided into sublocations and incorporated into another regression model (Supplemental Table 3 ). The estimated effect on the GCS score was -1.3 ( $\mathrm{p}=$ $0.009)$ for TAI in the splenium and -1.5 for TAI in the mesencephalon $(\mathrm{p}=0.004)$.

\section{Total Burden of TAI Lesions and GCS Scores}

The total number and volume of TAI lesions were significantly associated with the GCS score, whereas the total volume of contusions was not (Table 3). The volume of TAI on FLAIR and DWI provided better models for the GCS score than the number of TAI lesions on FLAIR, DWI, and T2*-weighted GRE/SWI (lower AIC and higher adjusted $\mathrm{R}^{2}$ ), both for simple and multiple regressions. The association of TAI lesion volume and GCS score was slightly stronger for DWI than FLAIR, but FLAIR vol- 


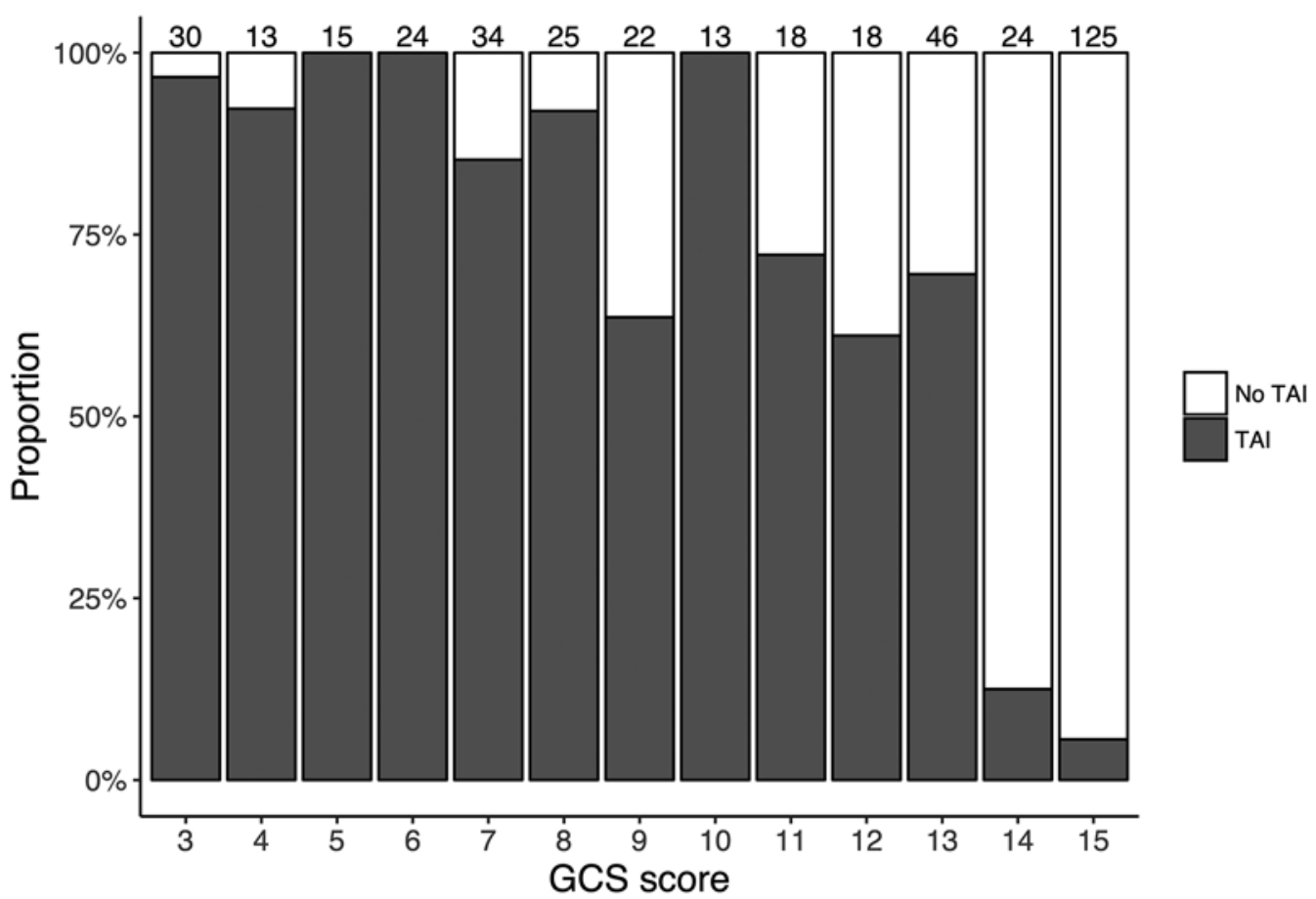

FIG. 2. The percentages of patients with TAI stratified by GCS score. Patients with mass lesions $(n=72)$ are not included in the figure. The total number of patients for each GCS score (regardless of MRI findings) is shown on top of the bars.

ume provided a better model fit. Using the volume of TAI on FLAIR for each brain region instead of the total volume did not improve the regression model fit (AIC 1382 vs 1377; Supplemental Table 4).

\section{TAI Lesions and Prolonged Duration of PTA}

TAI lesions were found in 34\% of patients with PTA for 0-7 days (96/286), 92\% with PTA for 8-27 days (81/88), and $96 \%$ with PTA for $>28$ days $(85 / 89, \mathrm{p}<0.001)$. Multiple logistic regression showed that bilateral TAI in the thalamus (OR 16.2) and in the brainstem (OR 4.2) led to the largest OR for PTA $>28$ days of all TAI findings (Ta- ble 4). Bilateral TAI in the thalamus and in the brainstem remained important predictors for prolonged PTA also after adjusting for the total TAI lesion burden (volume of TAI lesions on FLAIR). Among the different measures of total TAI lesion burden, the total volume of TAI on FLAIR and DWI had the strongest predictive value for PTA $>28$ days (Table 3 ). High ORs for PTA > 28 days were also found for Marshall CT scores 3-6 in patients with TAI (OR 9.3-12.3).

\section{Modified TAI-MRI Classification Scheme}

Based on the results of the regression analyses, TAI le-

TABLE 2. Relationship between the location of TAI lesions and the GCS score in patients with TAI

\begin{tabular}{|c|c|c|c|c|c|}
\hline \multirow[b]{2}{*}{ TAI Location } & \multirow{2}{*}{$\begin{array}{c}\text { No. of } \\
\text { Patients }\end{array}$} & \multicolumn{2}{|l|}{ Model I* } & \multicolumn{2}{|l|}{ Model II† } \\
\hline & & Estimate $(95 \% \mathrm{Cl})$ & $p$ Value & Estimate $(95 \% \mathrm{Cl})$ & $\mathrm{p}$ Value \\
\hline Hemispheres & 253 & 0.5 (-1.1 to 2.2$)$ & 0.525 & $0.9(-0.7$ to 2.6$)$ & 0.276 \\
\hline Corpus callosum & 144 & $-1.5(-2.3$ to -0.7$)$ & $<0.001$ & $-0.8(-1.7$ to 0.1$)$ & 0.070 \\
\hline Unilateral brainstem & 51 & $-1.6(-2.6$ to -0.6$)$ & 0.002 & $-1.4(-2.4$ to -0.4$)$ & 0.006 \\
\hline Bilateral brainstem & 32 & $-2.5(-3.8$ to -1.1$)$ & $<0.001$ & $-1.9(-3.3$ to -0.5$)$ & 0.007 \\
\hline Unilateral thalamus & 41 & $-0.3(-1.4$ to 0.9$)$ & 0.654 & $-0.1(-1.2$ to 1.0$)$ & 0.855 \\
\hline Bilateral thalamus & 20 & $-1.6(-3.2$ to 0.0$)$ & 0.051 & $-0.8(-2.5$ to 0.8$)$ & 0.319 \\
\hline Unilateral basal ganglia & 45 & $0.2(-0.8$ to 1.2$)$ & 0.699 & $0.3(-0.7$ to 1.3$)$ & 0.564 \\
\hline Bilateral basal ganglia & 15 & $-0.9(-2.7$ to 0.9$)$ & 0.340 & $-0.7(-2.5$ to 1.1$)$ & 0.457 \\
\hline Total vol of FLAIR-TAI & 229 & - & - & $-0.6(-0.9$ to -0.2$)$ & 0.001 \\
\hline
\end{tabular}

* Model I = multiple linear regression analysis adjusted for age, Marshall CT score, and alcohol intoxication. AIC = 1376; adjusted $\mathrm{R}^{2}=0.25$.

$\dagger$ Model II = model I + total volume of TAI on FLAIR. AIC $=1367$; adjusted $R^{2}=0.28$. 
TABLE 3. Relationship between the total burden of TAI lesions, GCS score, and prolonged duration of PTA

\begin{tabular}{|c|c|c|c|c|c|c|c|c|c|}
\hline & \multirow[b]{3}{*}{$\mathrm{r}_{\mathrm{s}}$} & \multicolumn{8}{|c|}{ Linear Regression for GCS Scores } \\
\hline & & \multicolumn{4}{|c|}{ Simple Regression Analysis } & \multicolumn{4}{|c|}{ Multiple Regression Analysis* } \\
\hline & & Estimate $(95 \% \mathrm{Cl})$ & $p$ Value & Adj $R^{2}$ & AIC & Estimate $(95 \% \mathrm{Cl})$ & $p$ Value & Adj $R^{2}$ & $\mathrm{AIC}$ \\
\hline \multicolumn{10}{|l|}{ TAl lesions† } \\
\hline No. on FLAIR & -0.27 & $-0.7(-1.2$ to -0.3$)$ & 0.001 & 0.06 & 1427 & $-0.7(-1.2$ to -0.3$)$ & 0.001 & 0.13 & 1412 \\
\hline Vol on FLAIR & -0.42 & $-0.9(-1.2$ to -0.7$)$ & $<0.001$ & 0.17 & 1392 & $-0.9(-1.2$ to -0.7$)$ & $<0.001$ & 0.23 & 1377 \\
\hline No. on DWI & -0.22 & $-0.7(-1.2$ to -0.2$)$ & 0.007 & 0.04 & 1433 & $-0.5(-1.0$ to 0.0$)$ & 0.059 & 0.10 & 1419 \\
\hline Vol on DWI & -0.32 & $-1.1(-1.4$ to -0.8$)$ & $<0.001$ & 0.15 & 1400 & $-1.0(-1.3$ to -0.6$)$ & $<0.001$ & 0.19 & 1391 \\
\hline No. on T2*-weighted GRE/SWI & -0.14 & $-0.5(-0.9$ to -0.2$)$ & 0.003 & 0.03 & 1436 & $-0.6(-1.0$ to -0.3$)$ & $<0.001$ & 0.10 & 1419 \\
\hline \multicolumn{10}{|l|}{ Contusionsł } \\
\hline \multirow[t]{4}{*}{ Vol of contusions } & 0.01 & $0.0(-0.3$ to 0.2$)$ & 0.754 & 0 & NA & $-0.1(-0.3$ to 0.2$)$ & 0.693 & 0.03 & NA \\
\hline & & \multicolumn{8}{|c|}{ Logistic Regression for PTA >28 Days } \\
\hline & & \multicolumn{4}{|c|}{ Simple Regression Analysis } & \multicolumn{4}{|c|}{ Multiple Regression Analysis* } \\
\hline & & OR $(95 \% \mathrm{Cl})$ & $p$ Value & Pseudo R² & $\mathrm{AIC}$ & OR $(95 \% \mathrm{Cl})$ & $p$ Value & Pseudo R2 & $\mathrm{AIC}$ \\
\hline \multicolumn{10}{|l|}{ TAl lesions† } \\
\hline No. on FLAIR & & 2.00 (1.47 to 2.79$)$ & $<0.001$ & 0.07 & 303 & 2.40 (1.67 to 3.55$)$ & $<0.001$ & 0.19 & 270 \\
\hline Vol on FLAIR & & $2.22(1.73$ to 2.94$)$ & $<0.001$ & 0.16 & 272 & 2.44 (1.84 to 3.37$)$ & $<0.001$ & 0.27 & 246 \\
\hline No. on DWI & & 2.10 (1.50 to 3.02$)$ & $<0.001$ & 0.07 & 303 & 2.30 (1.58 to 3.47$)$ & $<0.001$ & 0.18 & 274 \\
\hline Vol on DWI & & 2.68 (1.95 to 3.86$)$ & $<0.001$ & 0.16 & 273 & 2.79 (1.97 to 4.16$)$ & $<0.001$ & 0.25 & 251 \\
\hline No. on T2*-weighted GRE/SWI & & $1.52(1.20$ to 1.96$)$ & 0.001 & 0.04 & 312 & 1.88 (1.41 to 2.57$)$ & $<0.001$ & 0.18 & 275 \\
\hline \multicolumn{10}{|l|}{ Contusions } \\
\hline Vol of contusions & & $1.22(1.04$ to 1.44$)$ & 0.017 & 0.02 & NA & $1.12(0.93$ to 1.35$)$ & 0.226 & 0.07 & NA \\
\hline \multicolumn{10}{|c|}{ 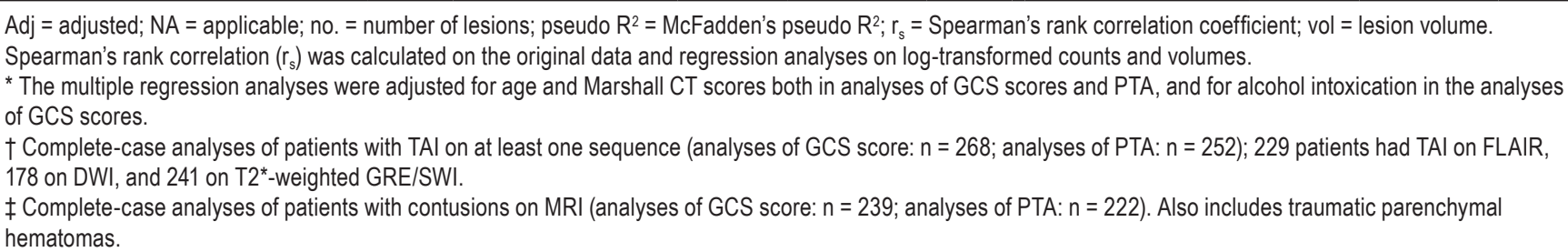 } \\
\hline
\end{tabular}

TABLE 4. Relationship between the location of TAl lesions and PTA duration $>28$ days in patients with TAl $(n=252)$

\begin{tabular}{|c|c|c|c|c|}
\hline \multirow[b]{2}{*}{ Variable } & \multicolumn{2}{|l|}{ Model I* } & \multicolumn{2}{|l|}{ Model II† } \\
\hline & OR $(95 \% \mathrm{Cl})$ & p Value & OR $(95 \% \mathrm{Cl})$ & $p$ Value \\
\hline Age & $1.02(1.00-1.05)$ & 0.022 & $1.03(1.00-1.05)$ & 0.019 \\
\hline \multicolumn{5}{|l|}{ Marshall CT score } \\
\hline 1 & Ref & NA & Ref & NA \\
\hline 2 & $2.41(0.70-11.65)$ & 0.207 & $2.21(0.59-11.41)$ & 0.279 \\
\hline 3 or 4 & $12.25(3.18-64.10)$ & $<0.001$ & $10.52(2.56-58.98)$ & 0.003 \\
\hline 5 or 6 & $9.28(2.51-47.21)$ & 0.002 & $10.89(2.65-61.57)$ & 0.002 \\
\hline TAl in hemispheres & $0.81(0.21-3.23)$ & 0.764 & $0.70(0.15-3.19)$ & 0.645 \\
\hline TAI in corpus callosum & $2.11(1.03-4.39)$ & 0.042 & $1.43(0.61-3.38)$ & 0.407 \\
\hline Unilateral TAI in brainstem & $1.98(0.88-4.46)$ & 0.097 & $2.11(0.89-5.01)$ & 0.087 \\
\hline Bilateral TAI in brainstem & $4.20(1.27-15.31)$ & 0.022 & $3.74(1.03-14.77)$ & 0.050 \\
\hline Unilateral TAl in thalamus & $2.14(0.87-5.27)$ & 0.095 & $1.75(0.68-4.47)$ & 0.242 \\
\hline Bilateral TAI in thalamus & $16.16(3.94-87.38)$ & $<0.001$ & $9.57(2.09-54.83)$ & 0.006 \\
\hline Unilateral TAI in basal ganglia & $0.62(0.24-1.54)$ & 0.317 & $0.50(0.18-1.30)$ & 0.168 \\
\hline Bilateral TAI in basal ganglia & $1.94(0.34-11.72)$ & 0.456 & $2.37(0.38-13.81)$ & 0.338 \\
\hline Total vol of FLAIR-TAI & & & $1.87(1.34-2.70)$ & $<0.001$ \\
\hline
\end{tabular}

${ }^{*}$ Model I = multiple logistic regression analysis. AIC $=252 ;$ pseudo $R^{2}=0.29$.

† Model II = model I + total volume of TAI on FLAIR. AIC = 239; pseudo $\mathrm{R}^{2}=0.34$. 

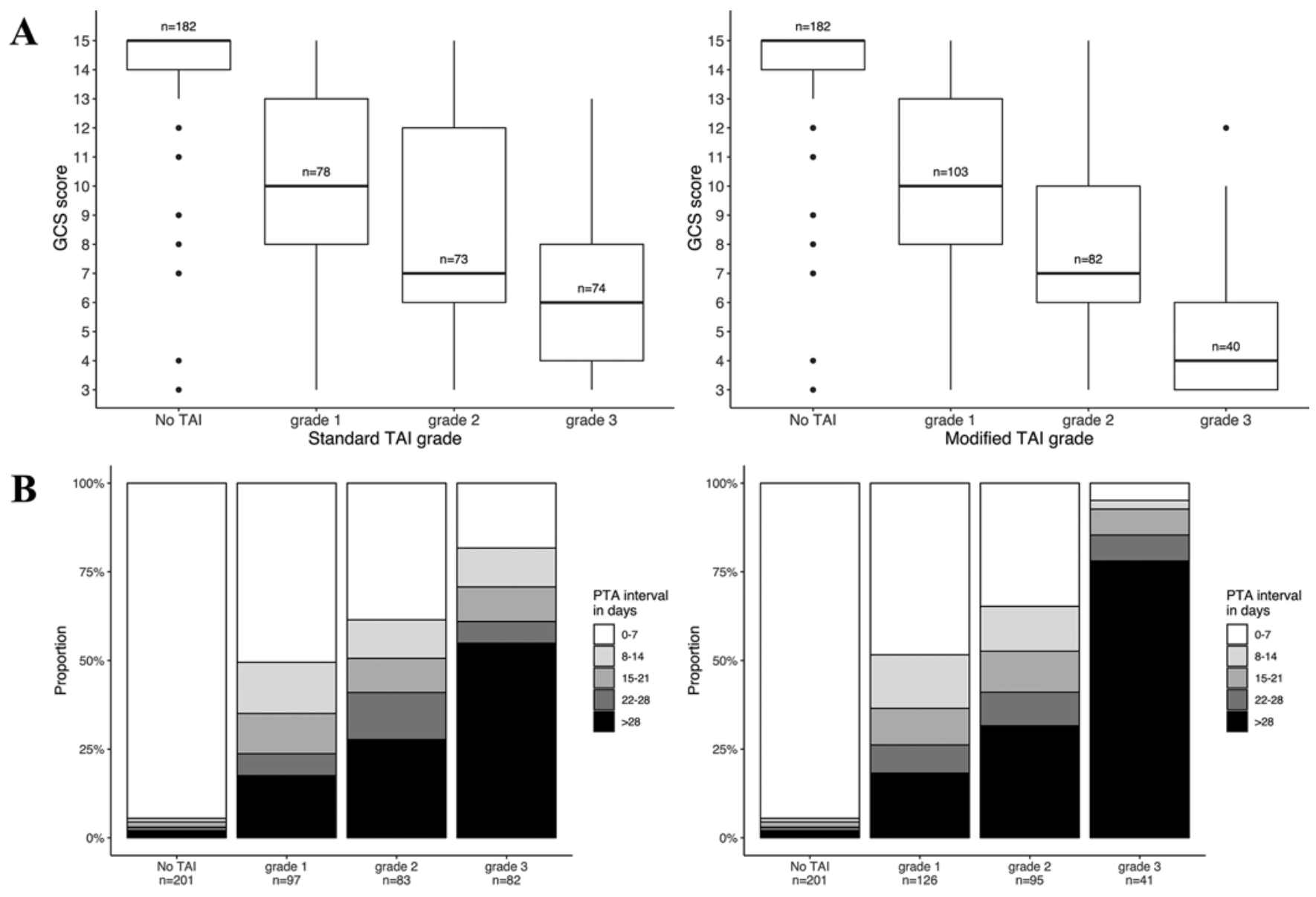

\begin{tabular}{lll}
\hline \multicolumn{3}{c}{ STANDARD TAI GRADE } \\
\hline TAI grade 1 & TAI grade 2 & TAI grade 3 \\
\hline $\begin{array}{l}\text { TAI in the } \\
\text { hemispheres or } \\
\text { cerebellum }\end{array}$ & $\begin{array}{l}\text { TAI in the corpus } \\
\text { callosum }\end{array}$ & $\begin{array}{l}\text { TAI in the } \\
\text { brainstem }\end{array}$ \\
\hline
\end{tabular}

\begin{tabular}{|c|c|c|c|}
\hline \multicolumn{4}{|c|}{ MODIFIED TAI GRADE } \\
\hline & ade 1 & Grade 2 & Grade 3 \\
\hline$\underline{1 \mathrm{~A}}$ & $\underline{1 \mathrm{~B}}$ & & \\
\hline $\begin{array}{l}\text { TAI in the } \\
\text { hemispheres or } \\
\text { cerebellum } \\
\text { (incl. unilateral } \\
\text { thalamus) }\end{array}$ & $\begin{array}{l}\text { TAI in the genu or } \\
\text { truncus of corpus } \\
\text { callosum }\end{array}$ & $\begin{array}{l}\text { Unilateral TAI in the } \\
\text { brainstem or TAI in } \\
\text { the splenium of } \\
\text { corpus callosum }\end{array}$ & $\begin{array}{l}\text { Bilateral TAI in the } \\
\text { brainstem or } \\
\text { thalamus }\end{array}$ \\
\hline
\end{tabular}

FIG. 3. The standard TAI grade compared with a modified TAI grade, their relation to the GCS score at the scene of injury or at admission (A), and their ability to discriminate between duration of posttraumatic amnesia intervals (B). Patients with mass lesions were excluded from the top two boxplots describing the relationship between the depth of lesions and the GCS score. incl. = including.

sions were ranked with regard to their relation to injury severity and presented as a possible modified TAI-MRI classification system (Fig. 3). ROC curves were constructed to visualize the ability of the multiple regression models, as well as the standard TAI grade and the modified TAI-MRI classification, to predict PTA $>28$ days (Fig. 4).

\section{Interrater Reliability}

The positive and negative agreement for the presence or absence of TAI were 0.90 (95\% CI 0.77-0.95) and 0.69 (95\% CI $0.42-0.84$ ), respectively. The ICC was 0.78 for the classification of TAI grade, 0.86 and 0.84 for the total volume of TAI on FLAIR and DWI, respectively, 0.88 for the total number of TAI on T2*-weighted GRE/SWI, and 0.85 for the volume of contusions.

\section{Discussion}

This large, prospective MRI study demonstrated that both the location and total burden of TAI lesions were associated with the level of consciousness at the scene of injury or at admission and prolonged duration of PTA. Bilateral TAI lesions in the brainstem was the MRI finding associated with the lowest GCS scores, followed by TAI lesions bilaterally in the thalamus, unilaterally in the brainstem, and lesions in the splenium of the corpus callosum. Patients with bilateral TAI in the thalamus had the highest odds ratios for having a prolonged duration of PTA, followed by bilateral TAI in the brainstem. The measures of total TAI lesion burden that were most strongly associated with both the GCS score and prolonged duration of PTA were the total volumes of TAI on FLAIR and DWI. 

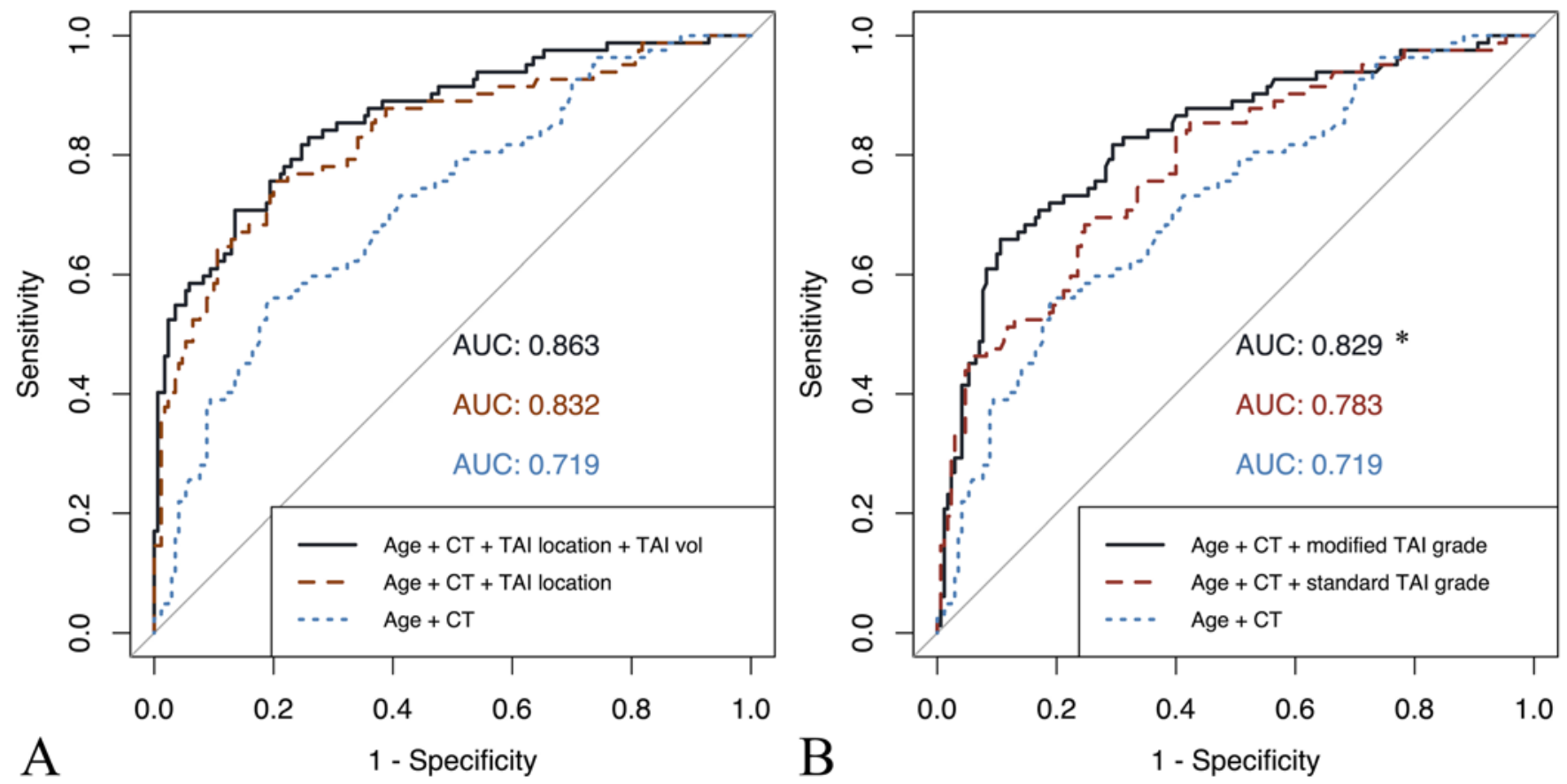

FIG. 4. ROC curves for different models predicting prolonged duration of PTA. A: The curves correspond to model I (red dashed curve) and model II (black solid curve) in Table 4. A curve representing a predictive model consisting of only age and Marshall CT score is presented for comparison (blue dotted curve). B: The curves correspond to the predictive value of the modified TAI grade (black solid curve) and the standard TAl grade (red dashed curve) for PTA > 28 days in multiple logistic regression models that also included age and Marshall CT score. A curve representing a predictive model containing only age and Marshall CT score is shown for comparison (blue dotted curve). *The model with the modified TAI-MRI grading system yielded a significantly larger AUC compared with the standard TAI grading system $(p=0.011)$.

\section{Location of TAI Lesions and the GCS Score}

We found that bilateral TAI lesions in the brainstem had the largest impact on the GCS score. However, bilateral thalamic lesions, unilateral brainstem lesions, and lesions in the splenium were also associated with lower GCS scores. Thus, the current study supports a severity gradient of TAI from superficial to deep lesions. ${ }^{25-28}$ Based on experimental work in pigs, and in a review of pathology and imaging studies, it has been argued that a decreased level of consciousness is due to the distribution of TAI lesions and especially affection of the brainstem rather than the total quantity of TAI lesions..$^{29,30}$ This hypothesis is partly supported by the current results, where the presence of TAI in the brainstem was associated with a large decrease in GCS score also after controlling for the total volume of TAI on FLAIR. On the other hand, the total volume of TAI on FLAIR also remained important for the GCS score after controlling for lesion location. Thus, a low GCS score at admission could, besides a significant mass lesion or general edema, suggest bilateral TAI in the brainstem, extensive TAI lesions throughout the brain, or all of the above.

\section{Total Burden of TAI Lesions and the GCS Score}

All the different measures of total TAI lesion burden were related to the GCS score, but the strongest associations were found for the volume of TAI on FLAIR and DWI. Previous studies have reported contradicting results concerning the relationship between the GCS score and the total number or volume of lesions on $\mathrm{T} 2 *$-weighted GRE $^{31-35}$ and FLAIR. ${ }^{35}$ Somewhat similar to our results, one of these studies reported that the volume of lesions on DWI had the strongest association with the GCS score..$^{34}$ However, these studies all had smaller sample sizes ( $\mathrm{s}$ 66) and either did not account for mass lesions and potential confounders or did not differentiate between contusions and TAI. In fact, the current study found little to no association between the volume of contusions on MRI and injury severity, demonstrating that such lesions and TAI lesions should be classified separately. Also, the strong correlation between the TAI volumes measured on FLAIR and DWI suggests that performing only FLAIR of the two could be sufficient in patients in whom clinical factors limit the feasibility of a more comprehensive MRI protocol.

\section{Location and Total Burden of TAI Lesions and Prolonged PTA}

Regardless of the total burden or presence of TAI elsewhere, bilateral TAI in the thalamus, followed by bilateral TAI in the brainstem, were the MRI findings that most strongly predicted prolonged PTA. Other important risk factors were mass lesions or general edema on CT (high Marshall CT scores). These findings represent novel insight into the reasons for prolonged PTA. The total burden of TAI lesions also significantly predicted prolonged PTA, and the relationship was strongest for the volume of TAI on FLAIR and DWI. Another TBI study $(n=65)$ found no 
correlation between PTA duration and the total number of TAI lesions on T2*-weighted GRE, while a correlation was found for the number of lesions in the corpus callosum and hippocampus. ${ }^{36}$ Supporting the current results, a study using diffusion tensor imaging in the chronic stage of TBI showed an association between longer duration of PTA and increased disruption of the microstructure in several white matter tracts and the thalamus. ${ }^{37}$ Parts of the thalamus are closely interconnected with the mammillary bodies and the hippocampus, and their integrity is likely critical for learning and memory. ${ }^{38}$ Moreover, strokes in certain thalamic regions are known to produce an amnestic syndrome similar to that of Korsakoff syndrome. ${ }^{38}$ Thus, high Marshall CT scores, and the quantity and distribution of TAI lesions on MRI, especially bilateral thalamic lesions, could serve as radiological biomarkers of patients at risk for prolonged PTA. Being able to predict a longer trajectory of recovery would be of great value to physicians when planning rehabilitation interventions and in guiding the expectations of patients and their families.

\section{Modified TAI-MRI Classification}

To summarize and simplify the relationship between TAI and injury severity described in Tables 2 and 4, we propose a first step toward a modified, pragmatic TAI classification system, where grades are ranked from the largest to smallest impact on injury severity:

3) Bilateral TAI in the brainstem and/or thalamus

2) Unilateral TAI in the brainstem and/or TAI in the splenium of corpus callosum

1) TAI in the

B) genu/truncus of corpus callosum

A) hemispheres/cerebellum (including unilateral thalamus lesions).

We emphasize that this suggestion is based on injury severity, where the change in TAI grade 3 is the most convincing result. However, this might be modified in followup studies, since the predictive value for outcome will be more important in clinical practice. The models that best explained variations in GCS scores and had the highest AUC for prediction of prolonged PTA included both TAI lesion location and total TAI lesion volume on FLAIR. However, manual estimation of lesion volumes on MRI is a time-consuming task. In the future, this process may be automated and performed by artificial intelligence technology. Until then, a location-based assessment of TAI lesions may be sufficient for the classification of injury, or even a surrogate marker for the total burden of lesions.

\section{Study Strengths and Limitations}

The prospective data collection is a notable strength of this study. Moreover, the large number of patients made it possible to perform multiple regression analyses to control for other factors affecting the GCS score and PTA duration, such as alcohol intoxication and mass lesions. Furthermore, MRI scans were read blinded for clinical information, using an internationally developed, comprehensive scoring template, which included manual estimations of the number and volume of lesions, even at a substructure level. To our knowledge, no previous studies have systematically mapped MRI lesions this thoroughly. The interrater agreement was excellent for the standard TAI grade and total TAI lesion burden. The early MRI in a cohort of this size is another strength of this study.

This study had some limitations. First, some selection bias will likely be present, as severely injured patients sometimes are not sufficiently stable to undergo early MRI, or even die before MRI can be performed. Second, a variety of MRI scanners were used, and the three different cohorts had somewhat differing MRI protocols. Also, most of the patients with moderate to severe TBI underwent MRI on a $1.5 \mathrm{~T}$ unit using T2*-weighted GRE, which has lower sensitivity to detect TAI lesions compared with 3T MRI ${ }^{39}$ and SWI ${ }^{40}$ which were used for almost all patients with mild TBI. Still, very few patients with mild TBI had MRI findings; thus, we consider the bias introduced as limited. Third, the time to MRI varied between patients, and lesions on FLAIR and DWI are known to attenuate over time. ${ }^{17}$ However, because MRI was performed later after severe TBI, the association between GCS scores and TAI lesions on FLAIR and DWI might have been even stronger had MRI been performed earlier. Finally, the use of validated scales for PTA assessment in all patients would have been preferable. Unfortunately, such tools are mostly used by rehabilitation professionals rather than in a neurosurgical ward. Additionally, intubation and sedation of TBI patients interferes with PTA assessment in the early phase. However, a cutoff for PTA of 28 days limited this problem.

\section{Conclusions}

This is one of the largest prospective studies of TBI patients with early MRI, and the first to use multivariable analyses in assessing the relationship between TAI lesions and injury severity. The current study demonstrated that bilateral TAI lesions in the brainstem were especially associated with low GCS scores. It also sheds novel light on the pathoanatomical basis of prolonged PTA after TBI, where bilateral TAI in the thalamus was found to be of particular importance. The total burden of TAI lesions, especially the volume of TAI lesions on FLAIR and DWI, were associated with both the GCS score at the scene of injury or at admission and late recovery of orientation and memory after TBI. This study presents a first step toward a modified, pragmatic classification system for TAI lesions on clinical MRI based on the lesion's relationship with injury severity, which includes bilateral lesions in the brainstem and thalamus as part of the grading. Such a system may serve as a guide for upcoming studies in the TAI-MRI project evaluating the importance of TAI lesion location and burden for patient outcome. A final modified classification scheme will be proposed after combining data from the ERA-NET TAI-MRI partners and the CENTER-TBI study.

\section{Acknowledgments}

We thank Torun Farnes and Berit Marianne Bjelkåsen for their contributions to the management of the patient databases. We would also like to thank Tone Jerstad for scoring the CT scans from the Oslo S-TBI cohort. Thanks to all collaborators in the 
ERA-NET NEURON TAI-MRI project for the development of the clinical MRI scoring template and for contributions during the drafting of the paper.

H.K.M. and K.G.M. received funding from the Norwegian University of Science and Technology (NTNU), ERA-NET NEURON, and the Research Council of Norway (TAI-MRI project). T.S. received a research grant during the study period from the liaison committee between the Central Norway Regional Health Authority and NTNU.

\section{References}

1. Gentry LR. Imaging of closed head injury. Radiology. 1994; 191(1):1-17.

2. Einarsen CE, Moen KG, Håberg AK, et al. Patients with mild traumatic brain injury recruited from both hospital and primary care settings: a controlled longitudinal magnetic resonance imaging study. J Neurotrauma. 2019;36(22):31723182.

3. Hill CS, Coleman MP, Menon DK. Traumatic axonal injury: mechanisms and translational opportunities. Trends Neurosci. 2016;39(5):311-324.

4. Skandsen T, Kvistad KA, Solheim O, et al. Prevalence and impact of diffuse axonal injury in patients with moderate and severe head injury: a cohort study of early magnetic resonance imaging findings and 1-year outcome. J Neurosurg. 2010;113(3):556-563.

5. Adams JH, Doyle D, Ford I, et al. Diffuse axonal injury in head injury: definition, diagnosis and grading. Histopathology. 1989;15(1):49-59.

6. Bramlett HM, Kraydieh S, Green EJ, Dietrich WD. Temporal and regional patterns of axonal damage following traumatic brain injury: a beta-amyloid precursor protein immunocytochemical study in rats. J Neuropathol Exp Neurol. 1997; 56(10):1132-1141.

7. Lifshitz J, Kelley BJ, Povlishock JT. Perisomatic thalamic axotomy after diffuse traumatic brain injury is associated with atrophy rather than cell death. J Neuropathol Exp Neurol. 2007;66(3):218-229.

8. Teasdale G, Maas A, Lecky F, et al. The Glasgow Coma Scale at 40 years: standing the test of time. Lancet Neurol. 2014;13(8):844-854.

9. Perel P, Arango M, Clayton T, et al. Predicting outcome after traumatic brain injury: practical prognostic models based on large cohort of international patients. BMJ. 2008;336(7641): 425-429.

10. Ponsford JL, Spitz G, McKenzie D. Using post-traumatic amnesia to predict outcome after traumatic brain injury. $J$ Neurotrauma. 2016;33(11):997-1004.

11. Marshman LAG, Jakabek D, Hennessy M, et al. Post-traumatic amnesia. J Clin Neurosci. 2013;20(11):1475-1481.

12. Maas AIR, Menon DK, Adelson PD, et al. Traumatic brain injury: integrated approaches to improve prevention, clinical care, and research. Lancet Neurol. 2017;16(12):987-1048.

13. Saatman KE, Duhaime A-C, Bullock R, et al. Classification of traumatic brain injury for targeted therapies. J Neurotrauma. 2008;25(7):719-738.

14. Skandsen T, Einarsen CE, Normann I, et al. The epidemiology of mild traumatic brain injury: the Trondheim MTBI follow-up study. Scand J Trauma Resusc Emerg Med. 2018; 26(1):34.

15. Røe C, Skandsen T, Anke A, et al. Severe traumatic brain injury in Norway: impact of age on outcome. J Rehabil Med. 2013;45(8):734-740.

16. de Leeuw FE, de Groot JC, Achten E, et al. Prevalence of cerebral white matter lesions in elderly people: a population based magnetic resonance imaging study. The Rotterdam Scan Study. J Neurol Neurosurg Psychiatry. 2001;70(1):9-14.

17. Moen KG, Skandsen T, Folvik M, et al. A longitudinal MRI study of traumatic axonal injury in patients with moderate and severe traumatic brain injury. J Neurol Neurosurg Psychiatry. 2012;83(12):1193-1200.

18. Maas AIR, Menon DK, Steyerberg EW, et al. Collaborative European Neurotrauma Effectiveness Research in Traumatic Brain Injury (CENTER-TBI): a prospective longitudinal observational study. Neurosurgery. 2015;76(1):67-80.

19. Novack TA, Dowler RN, Bush BA, et al. Validity of the orientation log, relative to the Galveston Orientation and Amnesia Test. J Head Trauma Rehabil. 2000;15(3):957-961.

20. Marshall LF, Marshall SB, Klauber MR, et al. A new classification of head injury based on computerized tomography. $J$ Neurosurg. 1991;75(suppl):S14-S20.

21. Griffanti L, Jenkinson M, Suri S, et al. Classification and characterization of periventricular and deep white matter hyperintensities on MRI: a study in older adults. Neuroimage. 2018;170:174-181.

22. Walker WC, Ketchum JM, Marwitz JH, et al. A multicentre study on the clinical utility of post-traumatic amnesia duration in predicting global outcome after moderate-severe traumatic brain injury. J Neurol Neurosurg Psychiatry. 2010; 81(1):87-89.

23. de Vet HCW, Dikmans RE, Eekhout I. Specific agreement on dichotomous outcomes can be calculated for more than two raters. J Clin Epidemiol. 2017;83:85-89.

24. Hallgren KA. Computing inter-rater reliability for observational data: an overview and tutorial. Tutor Quant Methods Psychol. 2012;8(1):23-34.

25. Moe HK, Limandvik Myhr J, Moen KG, et al. Association of cause of injury and traumatic axonal injury: a clinical MRI study of moderate and severe traumatic brain injury. J Neurosurg. 2020;133(5):1559-1567.

26. Grados MA, Slomine BS, Gerring JP, et al. Depth of lesion model in children and adolescents with moderate to severe traumatic brain injury: use of SPGR MRI to predict severity and outcome. J Neurol Neurosurg Psychiatry. 2001;70(3): 350-358.

27. Ommaya AK, Gennarelli TA. Cerebral concussion and traumatic unconsciousness. Correlation of experimental and clinical observations of blunt head injuries. Brain. 1974;97(4): 633-654.

28. Moe HK, Moen KG, Skandsen T, et al. The influence of traumatic axonal injury in thalamus and brainstem on level of consciousness at scene or admission: a clinical magnetic resonance imaging study. J Neurotrauma. 2018;35(7):975984.

29. Smith DH, Nonaka M, Miller R, et al. Immediate coma following inertial brain injury dependent on axonal damage in the brainstem. J Neurosurg. 2000;93(2):315-322.

30. Rosenblum WI. Immediate, irreversible, posttraumatic coma: a review indicating that bilateral brainstem injury rather than widespread hemispheric damage is essential for its production. J Neuropathol Exp Neurol. 2015;74(3):198-202.

31. Tong KA, Ashwal S, Holshouser BA, et al. Diffuse axonal injury in children: clinical correlation with hemorrhagic lesions. Ann Neurol. 2004;56(1):36-50.

32. Scheid R, Preul C, Gruber O, et al. Diffuse axonal injury associated with chronic traumatic brain injury: evidence from T2*-weighted gradient-echo imaging at 3 T. AJNR Am J Neuroradiol. 2003;24(6):1049-1056.

33. Yanagawa Y, Tsushima Y, Tokumaru A, et al. A quantitative analysis of head injury using $\mathrm{T} 2 *$-weighted gradient-echo imaging. J Trauma. 2000;49(2):272-277.

34. Schaefer PW, Huisman TAGM, Sorensen AG, et al. Diffusion-weighted MR imaging in closed head injury: high correlation with initial Glasgow Coma Scale score and score on modified Rankin Scale at discharge. Radiology. 2004;233(1): $58-66$.

35. Pierallini A, Pantano P, Fantozzi LM, et al. Correlation be- 
tween MRI findings and long-term outcome in patients with severe brain trauma. Neuroradiology. 2000;42(12):860-867.

36. Mazwi NL, Izzy S, Tan CO, et al. Traumatic microbleeds in the hippocampus and corpus callosum predict duration of posttraumatic amnesia. J Head Trauma Rehabil. 2019;34(6): E10-E18.

37. Håberg AK, Olsen A, Moen KG, et al. White matter microstructure in chronic moderate-to-severe traumatic brain injury: impact of acute-phase injury-related variables and associations with outcome measures. J Neurosci Res. 2015; 93(7):1109-1126.

38. Schmahmann JD. Vascular syndromes of the thalamus. Stroke. 2003;34(9):2264-2278.

39. Scheid R, Ott DV, Roth $\mathrm{H}$, et al. Comparative magnetic resonance imaging at 1.5 and 3 Tesla for the evaluation of traumatic microbleeds. J Neurotrauma. 2007;24(12):1811-1816.

40. Geurts BHJ, Andriessen TMJC, Goraj BM, Vos PE. The reliability of magnetic resonance imaging in traumatic brain injury lesion detection. Brain Inj. 2012;26(12):1439-1450.

\section{Disclosures}

The authors report no conflict of interest concerning the materials or methods used in this study or the findings specified in this paper.

\section{Author Contributions}

Conception and design: Moen, Moe, Follestad, Vik. Acquisition of data: Moen, Moe, Andelic, Håberg, Flusund, Kvistad, Saksvoll, Olsen, Abel-Grüner, Skandsen, Vik. Analysis and interpretation of data: Moen, Moe, Follestad, Håberg, Skandsen, Vik. Drafting the article: Moe. Critically revising the article: Moen, Moe, Follestad, Andelic, Håberg, Flusund, Saksvoll, Olsen, Abel-Grüner, Sandrød, Skandsen, Vik. Statistical analysis: Moe, Follestad. Administrative/technical/material support: Moe, Sandrød. Study supervision: Moen, Vik.

\section{Supplemental Information}

\section{Online-Only Content}

Supplemental material is available with the online version of the article.

Supplemental Text and Tables. https://thejns.org/doi/suppl/ 10.3171/2020.6.JNS20112.

\section{Previous Presentations}

Portions of this work were presented in poster form at the 2 nd Nordic Neurotrauma Conference (NNC), November 18-20, 2019, in Lund, Sweden, and at the ERANET NEURON Mid-Term Symposium, January 21-23, 2019, in Bonn, Germany.

\section{Correspondence}

Kent Gøran Moen: Norwegian University of Science and Technology (NTNU), Trondheim, Norway. kent.g.moen@ntnu.no. 